\section{Cardiovascular risk factors in newly diagnosed abnormal glucose tolerance: comparison of 1997 ADA and 1985 WHO criteria}

Dear Sir,

From a public health perspective, diabetes diagnostic criteria should identify the part of a population with an increased risk for micro- and macrovascular complications. We have compared the 1997 ADA criteria with the 1985 WHO criteria with respect to prevalence of abnormal glucose tolerance (diabetes and impaired glucose tolerance (IGT) according to WHO; diabetes and impaired fasting glucose (IFG) according to ADA) and its relation with cardiovascular risk factors [1,2].

Fasting oral glucose tolerance tests according to WHO were carried out in 216 former participants of the populationbased study: Monitoring trends and determinants in cardiovascular disease (MONICA) survey in Augsburg (Germany) (mean age: $65.5 \pm 9.0$ years, range: $32-83$ years; men: $57 \%$ ) without previously known diabetes [2]. Subjects were recruited (response rate $64 \%$ ) and investigated as part of a cross-sectional study on cardiovascular and other complications in diabetic and non-diabetic patients: Kooperative Gesundheitsforschung in der Region Augsburg (cooperative health research in the Augsburg region) (KORA-A).

Table 1. Clinical and metabolic characteristics of subjects with newly diagnosed diabetes or impaired glucose tolerance (IGT) according to WHO (OGTT) and diabetes or impaired
There was neither a difference in the overall prevalence of diabetes in this population according to both criteria (WHO: $n=10 ; 4.6 \%, 95 \%$ CI: $2.2-8.3 \%$; ADA: $n=14 ; 6.5 \%, 3.5-$ $10.6 \%)$, nor in the prevalence of IGT $(n=50 ; 23 \%, 18-29 \%)$ and IFG $(n=53 ; 25 \%, 19-31 \%)(p=0.24$, test for symmetry). The overall kappa statistic, however, was 0.39 (95\% CI: $0.19-0.49)$ indicating relatively poor agreement (kappa statistic: $>0.75$ good agreement; $<0.40$ poor agreement) between the two diagnostic criteria for abnormal glucose tolerance [3].

Cardiovascular risk factors were assessed after stratifying subjects into four groups:

1. abnormal glucose tolerance (diabetes or IGT) according to WHO and normal glucose tolerance according to ADA,

2. abnormal glucose tolerance (diabetes or IFG) according to ADA and normal glucose tolerance according to WHO,

3. abnormal glucose tolerance according to both criteria,

4. normal glucose tolerance according to both ADA and WHO criteria.

The characteristics and cardiovascular risk factors of these four groups are given in Table 1. Age, BMI, fasting and 2-h plasma glucose, triglycerides, uric acid, and albuminuria. (random urine albumin concentration) were considerably higher for subjects classified as having abnormal glucose tolerance according to WHO alone (group 1: all IGT) compared with nondiabetic subjects according to both criteria (group 4) $(p<0.05)$. Prevalence of microalbuminuria (albumin-

fasting glucose (IFG) according to ADA criteria among 216 former MONICA survey participants

\begin{tabular}{|c|c|c|c|c|}
\hline & $\begin{array}{l}\text { Abnormal GT } \\
(\text { WHO) and } \\
\text { normal GT } \\
(\mathrm{ADA})^{\mathrm{a}}\end{array}$ & $\begin{array}{l}\text { Abnormal GT } \\
\text { (ADA) and } \\
\text { normal GT } \\
(\mathrm{WHO})^{\mathrm{b}}\end{array}$ & $\begin{array}{l}\text { Abnormal GT } \\
(\text { WHO) and } \\
\text { abnormal GT } \\
(\mathrm{ADA})^{\mathrm{c}}\end{array}$ & $\begin{array}{l}\text { Normal GT } \\
\text { (WHO) and } \\
\text { normal GT } \\
(\text { ADA })^{d}\end{array}$ \\
\hline$n$ & 25 & 32 & 35 & 124 \\
\hline New diabetes/IGT or IFG $(n)$ & $0 / 25$ & $4 / 28$ & $6 / 29$ & $0 / 0$ \\
\hline Age (years) & $69 \pm 9^{\mathrm{e}}$ & $66 \pm 8$ & $68 \pm 9^{\mathrm{e}}$ & $64 \pm 9$ \\
\hline Waist-to-hip ratio & $0.93 \pm 0.08$ & $0.94 \pm 0.11$ & $0.95 \pm 0.08^{\mathrm{e}}$ & $0.92 \pm 0.08$ \\
\hline $\mathrm{FPG}(\mathrm{mmol} / \mathrm{l})$ & $5.6 \pm 0.3^{\mathrm{e}}$ & $6.5 \pm 0.4^{\mathrm{e}}$ & $7.5 \pm 2.8^{\mathrm{e}}$ & $5.3 \pm 0.4$ \\
\hline 2-h PG (mmol/l) & $8.8 \pm 0.8^{\mathrm{e}}$ & $5.9 \pm 1.1$ & $10.4 \pm 3.4^{\mathrm{e}}$ & $5.6 \pm 1.1$ \\
\hline $\mathrm{HbA}_{1 \mathrm{c}}(\%)$ & $5.1 \pm 0.4$ & $5.4 \pm 0.7^{\mathrm{e}}$ & $5.6 \pm 1.2^{\mathrm{e}}$ & $5.0 \pm 0.4$ \\
\hline Systolic BP (mm Hg) & $144 \pm 21$ & $135 \pm 21$ & $147 \pm 19^{\mathrm{e}}$ & $137 \pm 23$ \\
\hline Diastolic BP (mm Hg) & $78 \pm 11$ & $78 \pm 11$ & $81 \pm 12^{\mathrm{e}}$ & $77 \pm 12$ \\
\hline Cholesterol $(\mathrm{mmol} / \mathrm{l})$ & $5.8 \pm 1.1$ & $6.0 \pm 0.8$ & $6.0 \pm 0.9$ & $5.9 \pm 1.0$ \\
\hline Uric acid $(\mu \mathrm{mol} / \mathrm{l})$ & $369 \pm 89^{e}$ & $351 \pm 71^{\mathrm{e}}$ & $369 \pm 83^{\mathrm{e}}$ & $315 \pm 83$ \\
\hline Albuminuria (mg/l) & $12(1-168)^{\mathrm{e}}$ & $12(1-642)^{\mathrm{e}}$ & $15(1-387)^{\mathrm{e}}$ & $6(1-1259)$ \\
\hline \multicolumn{5}{|c|}{$\begin{array}{l}\text { GT: Glucose tolerance; FPG: fasting plasma glucose; } 2-\mathrm{h} \text { PG: } \\
2 \text { hour plasma glucose (OGTT) } \\
\text { Data are mean } \pm \text { SD, or median and range (triglycerides, crea- } \\
\text { tinine, albuminuria) } \\
\text { a Diabetes or IGT (WHO) and normal fasting glucose (ADA) } \\
\text { b Diabetes or IFG (ADA) and normal glucose tolerance }\end{array}$} \\
\hline
\end{tabular}
(WHO)

Corresponding author: Dr. W. Rathmann MSPH, Diabetes Research Institute, Auf'm Hennekamp 65, D-40225 Düsseldorf, Germany 
uria $\geq 20 \mathrm{mg} / \mathrm{l}$ ) was also significantly increased among subjects in group 1 than in the non-diabetic group 4 (36\% vs $16 \%$; $p<0.05)$. Applying the ADA criteria, this first group would have been categorized as having normal glucose tolerance.

On the other hand, 32 subjects (4 with diabetes, 28 with IFG), who had abnormal glucose tolerance according to ADA criteria alone (group 2), were also on average more obese, had higher fasting and 2-h plasma glucose, $\mathrm{HbA}_{1 \mathrm{c}}$ triglycerides, uric acid and albuminuria than the non-diabetic group $4(p<0.05)$. Microalbuminuria was not significantly more prevalent than in the non-diabetic group (28\% vs $16 \%$, Fischer's exact test: $p=0.13$ ).

Thus, applying either 1997 ADA or 1985 WHO criteria, a considerable number of subjects with an adverse cardiovascular risk factor profile would be missed. Microalbuminuria, a powerful predictor of cardiovascular morbidity and mortality, was more prevalent in subjects classified as IGT according to WHO but who had normal fasting glucose according to ADA. Our results based on a small selected population are in line with a recent analysis of the Hoorn Study, a large populationbased survey from the Netherlands [4]. These data suggest, that if the fasting threshold for diabetes diagnosis falls as proposed by ADA, the use of the 2-h glucose concentration should be maintained to identify people with IGT (WHO). This may offer the "best of two worlds" [5].

Yours sincerely,

W. Rathmann, G. Giani, A. Mielck

\section{References}

1. The Expert Committee on the Diagnosis and Classification of Diabetes Mellitus (1997) Report of the Expert Committee on the Diagnosis and Classification of Diabetes Mellitus. Diabetes Care 20: 1183-1197

2. World Health Organization Study Group on Diabetes Mellitus (1985) Technical report series No 727, WHO, Geneva

3. Fleiss JL (1981) Statistical methods for rates and proportions, 2nd edn. Wiley, New York Chichester Brisbane Toronto Singapore

4. De Vegt F, Dekker JM, Stehouwer CDA, Nijpels G, Boutet M, Heine RJ (1998) The 1997 American Diabetes Association criteria versus the 1985 World Health Organization criteria for the diagnosis of abnormal glucose tolerance. Diabetes Care 21: 1686-1690

5. Davies MJ (1998) A change in the diagnostic criteria for diabetes mellitus: what will the impact be? Practical Diabetes International 15: 227-229

\title{
Erratum
}

\section{Intramyocellular lipid concentrations are correlated with insulin sensitivity in humans: a ${ }^{\mathbf{1}} \mathbf{H}$ NMR spectroscopy study (Rapid communication)}

\author{
M.Krssak ${ }^{1}$, K.Falk Petersen ${ }^{1}$, A. Dresner ${ }^{1}$, L.DiPietro ${ }^{2}$, S. M. Vogel ${ }^{1}$, D. L. Rothman ${ }^{1}$, G. I.Shulman ${ }^{3}$, M. Roden ${ }^{4}$ \\ ${ }^{1}$ Department of Internal Medicine Yale University School of Medicine, New Haven, Connecticut, USA \\ ${ }^{2}$ The John B. Pierce Laboratory and Department of Epidemiology \& Public Health Yale University School of Medicine, \\ New Haven, Connecticut, USA \\ ${ }^{3}$ Howard Hughes Medical Institute, Yale University School of Medicine, New Haven, Connecticut, USA \\ ${ }^{4}$ Division of Endocrinology and Metabolism, Department of Internal Medicine III, University of Vienna, Vienna, Austria
}

Diabetologia (1999) 42: 113-116

\begin{abstract}
Sentence 5 should read:
Simple linear regression analysis showed an inverse correlation $(r=-0.579, p=0.0037)$ between intramyocellular lipid content and M-value (100-120 min of clamp) as well as between fasting plasma non-esterified fatty acids concentration and M-value $(r=-0.54, p=0.0267)$.
\end{abstract}

This change also applies to the Results Section (paragraph 2, sentence 2) and the last sentence of the legend to Figure 1.

Table 1. Last line is to read:
IMCL content (\% of water resonance peak intensity)
$1.28 \pm 0.06$
$0.74-1.76$

These corrections are due to an incorrect $\mathrm{T} 1$ and $\mathrm{T} 2$ relaxation correction of IMCL content data and do not change the conclusions of the paper. 